\title{
Influence of Phase Differences in Beam Shaping of Multiple Laser Diodes Using a Kinoform
}

\author{
Yosuke Kogaa,, ${ }^{\mathrm{a},}$ Yuki Misaki ${ }^{\mathrm{a}}$, Shiyuan Yang ${ }^{\mathrm{a}}$ \\ ${ }^{a}$ Kyushu Institute of Technology, 1-1 Sensui-cho Tobata-ku Kitakyushu city, Fukuoka, 804-8550, Japan
}

*Corresponding Author: o349412y@mail.kyutech.jp

\begin{abstract}
Laser diodes have advantages of compact size, higher luminous efficacy and long-life. However disadvantage of laser diode is low output light power compared to other lasers. It is necessary to use multiple laser diodes for the applications of laser machining, laser marking. However there is no method to shape multiple laser lights. This paper proposes the design method for beam shaping of multiple laser diodes and describes the influence of shaped beam intensity on the number of laser diodes, irradiation area ratio to Kinoform size and sampling points of the focusing area.
\end{abstract}

Keywords: Kinoform, Multiple laser diodes, Beam shaping.

\section{Introduction}

Laser diode has been used in various applications because of its advantages of compact size, higher luminous efficacy and long-life. However the disadvantage of laser diode is low output light power compared to other lasers. It is necessary to use multiple laser diodes for the applications of laser machining, laser marking and etc. In addition, beam shaping of output light is necessary to increase the quality of application. Kinoform is a phase only CGH that has high illuminating light efficiency than other computer-generated holograms, Kinoform is powerful for beam shaping of laser light, but there is no method to shape multiple laser lights because the phase differences of laser lights are not determined at every power-on time. In this study, we suggest a design method for the beam shaping of multiple laser lights of Kinoform using band-unlimited reconstruction to relax the influence of phase differences of laser lights. We first show the influence of phase differences of laser sources in band-

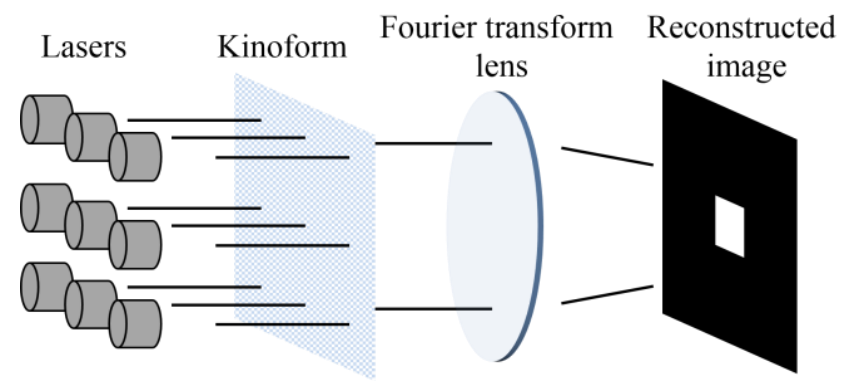

Fig. 1. Fourier transformation type

limited reconstruction Kinoform and band-unlimited one. After that, we show the influence of phase differences of the parameters of Kinoform using band-unlimited.

\section{Principle of Kinoform}

Reconstructing of Kinoform is used Fresnel diffraction or Fraunhofer diffraction depending on the distance. Fraunhofer diffraction can be approximated to Fourier diffraction. Figure 1 shows Fourier transformation type Kinoform playback optical system. Fig.1Phase differences of multiple laser lights are 0 when Kinoform is generated. Two-dimensional intensity distribution of the original picture image is $N \times N$ [pixel] according to sampling theorem. Input image adding the dummy area of initial value 0 to the surroundings of an original image is data of $M \times M$ [pixel] represented by $f(m, n)$, when Kinoform is generating. Figure 2 shows the dummy area. The initial phase distribution of original image is random phase. Fourier inverse transform of the $f(m, n)$ is represented by the equ.1. The Kinoform distribution is represented $W(k, l)$ by replacing the amplitude value $F(k, l)$ of the Fourier components with the constant value. The Kinoform distribution $W(k, l)$ is represented by the equ.2.Intensity distribution of the reconstructed image is 


$$
\begin{aligned}
F(k, l) & =\frac{1}{N^{2}} \sum_{x=-\frac{N}{2}}^{\frac{N}{2}-1} \sum_{y=-\frac{N}{2}}^{\frac{N}{2}-1} f(m, n) \exp \left[\frac{j 2 \pi}{N}(m k+n l)\right] \\
& =|F(k, l)| \exp [j \varphi(k, l)]
\end{aligned}
$$

$W(k, l)=A \exp [j \varphi(k, l)]$

$\left|f^{\prime}(m, n)\right|=\mid \sum_{x=-\frac{N}{2}}^{\frac{N}{2}-1} \sum_{y=-\frac{N}{2}}^{\frac{N}{2}-1}\left\{G_{s}(k, l)\right.$

$$
\left.\times W(k, l) \exp \left[-\frac{j 2 \pi}{N}(m k+n l)\right]\right\}\left.\right|^{2}
$$

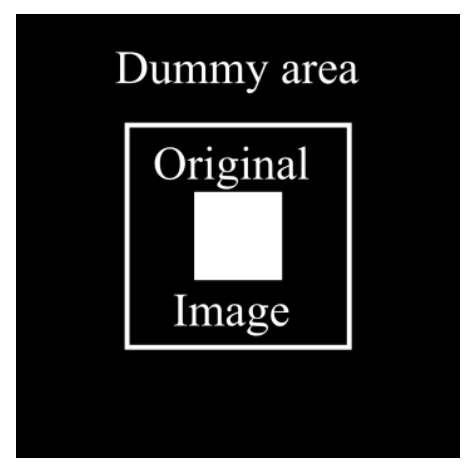

Fig.2. Dummy area

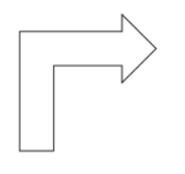

Restriction condition on the kinoform side

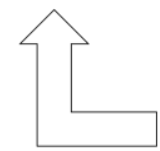

\section{DFT}

Fig.3. iterative dummy area method

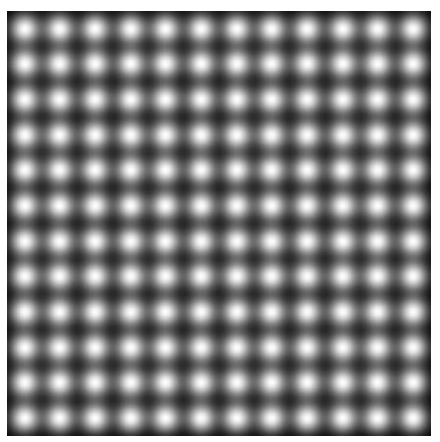

Fig.4. Relationship of the multiple laser lights represented by the equ.3. $G_{s}(k, l)$ represents the Gaussian distribution of the laser beam. One of the method to reduce the reconstructed image error is iterative dummy area method. Iterative dummy area method is the method using the amplitude flexibility and phase flexibility of dummy area and phase flexibility of original image. It is possible to reduce the reconstructed image error by iteration using the amplitude flexibility and phase flexibility. The restriction condition on Kinoform side at the time of iteration is to replace the obtained amplitude distribution to the amplitude distribution of the multiple laser. The restriction condition on the reconstructed image side at the time of iteration is to replace the obtained amplitude distribution to the amplitude distribution of the original image. Figure 3 shows the flow of iterative dummy area method.

\section{Simulation and results}

\subsection{Simulation method}

Multiple laser lights are placed evenly in square in this simulate. The Resolution width of general optical phase modulator is approximately $13[\mu \mathrm{m}]$. Gaussian radius of general laser diode is approximately $0.15[\mathrm{~mm}]$. Therefore Gaussian radius of laser diode is 12 [pixel] in this simulation. Dummy area is $N \times N$ [pixel]. Kinoform is $M \times M$ [pixel]. Number of lasers is $A \times A$. In the case of this simulation, distance between the centers of lasers is represented by the following equation.

$$
T=M / A
$$

Irradiated area ratio to Kinoform represent the extent of the laser lights irradiating to Kinoform. Irradiated area ratio to Kinoform is represented " $\mathrm{S}$ ".

$$
S=\frac{12^{2} \pi A^{2}}{N^{2}}
$$

Figure 4 shows the positional relationship of the multiple laser lights. We used 5 original images which is shown fig.5 in this simulation. Table 1 shows sampling points of all area and sampling points of focusing area.

Table1: original image size

\begin{tabular}{|l|l|l|}
\hline & All area & Focusing area \\
\hline Fig.5(a) & $64 \times 64$ [pixel] & $32 \times 32$ [pixel] \\
\hline Fig.5(b) & $128 \times 128$ [pixel] & $32 \times 32[$ pixel] \\
\hline Fig.5(c) & $256 \times 256[$ pixel] & $32 \times 32$ [pixel] \\
\hline Fig.5(d) & $256 \times 256[$ pixel] & $64 \times 64$ [pixel] \\
\hline Fig.5(e) & $256 \times 256[$ pixel] & $128 \times 128$ [pixel] \\
\hline
\end{tabular}




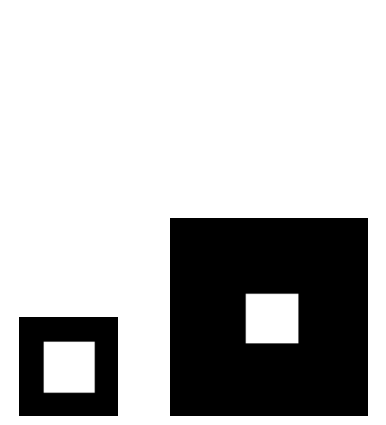

(b)

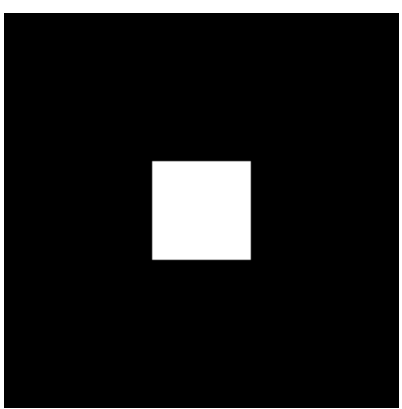

(d)

Fig.5. original images

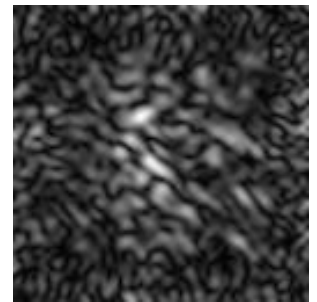

(A-1)band-limited

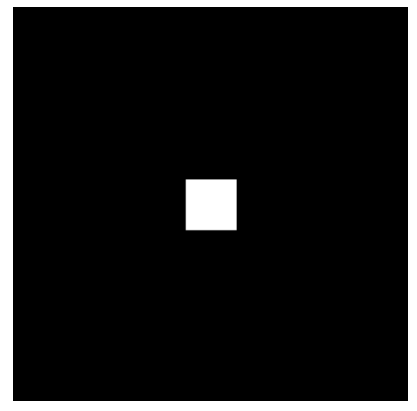

(c)

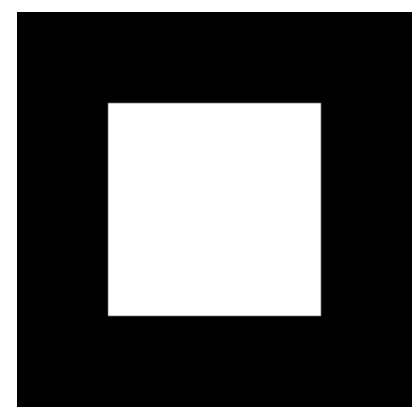

(e)

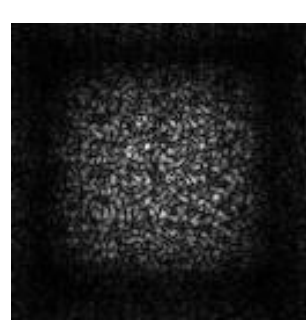

(A-2)band-unlimited
Fig.6. simulation A result

Table2: Simulation A condition

\begin{tabular}{|l|c|c|}
\hline & A-1 & A-2 \\
\hline Original Image & \multicolumn{2}{|c|}{ fig.5(a) } \\
\hline Dummy area ratio & $16 \times 16$ & \multicolumn{2}{|c|}{$512 \times 512$ [pixel] } \\
\hline Kinoform & \multicolumn{2}{|c|}{$12 \times 12$} \\
\hline Number of lasers & \multicolumn{2}{|c|}{1000 times } \\
\hline Number of iteration & \multicolumn{2}{|c|}{0.249} \\
\hline $\begin{array}{l}\text { Irradiation area / } \\
\text { Kinoform size }\end{array}$ & $1 / 2$ \\
\hline Band-limited & \multicolumn{2}{|c|}{-} \\
\hline
\end{tabular}

\subsection{Comparison of Band-limited and Band-unlimited}

First we verify the influence of phase differences on beam shaping of multiple laser lights in the case of bandlimited and band-unlimited. Table2 shows simulation A condition of Kinoform generation. Figure 6 shows the reconstructed images of Kinoform of simulation $\mathrm{A}$ by multiple laser lights with phase difference. Figure 6 is the reconstructed images of the original image area only. Figure 6 shows that beam shaping of Kinoform using bandunlimited is better than beam shaping of kinofrom using band-limited. In the case of using band-limited, Kinoform is satisfied sampling theorem. However the errors is big because phase distribution satisfying sampling theorem is added random phase difference. In the case of using bandunlimited, Kinoform is not satisfied sampling theorem. Therefore the value between discrete points is random. In the case random value between discrete points is added the value of random phase difference, the value between discrete points is random. Therefore influence of phase difference is relaxed. In the case of application of laser machining, the problem of discrete image as shown in Fig. 6(A-2) can be ignored for thermal dispersion. Next we verify the influence of parameters of Kinoform using band-unlimited on beam shaping of multiple laser lights.

\subsection{Verification of parameters}

We verify the influence of the number of lasers. Table3 shows simulation $\mathrm{B}$ condition of Kinoform generation. Figure 7 shows the reconstructed images of Kinoform of simulation B by multiple laser lights with phase difference. Figure 7 is the reconstructed images of the original image area only.

Table3: Simulation B condition

\begin{tabular}{|l|c|c|c|}
\hline & B-1 & B-2 & B-3 \\
\hline Original Image & \multicolumn{3}{|c|}{ Fig.5(a) } \\
\hline $\begin{array}{l}\text { Dummy area } \\
\text { ratio }\end{array}$ & $8 \times 8$ & $16 \times 16$ & $32 \times 32$ \\
\hline Kinoform & $\begin{array}{c}512 \times \\
512[\mathrm{pixel}]\end{array}$ & $\begin{array}{c}1024 \times \\
1024[\mathrm{pixel}]\end{array}$ & $\begin{array}{c}2048 \times \\
2048[\mathrm{pixel}]\end{array}$ \\
\hline $\begin{array}{l}\text { Number of } \\
\text { lasers }\end{array}$ & $12 \times 12$ & $24 \times 24$ & $48 \times 48$ \\
\hline $\begin{array}{l}\text { Number of } \\
\text { iteration }\end{array}$ & 1000 & \\
\hline $\begin{array}{l}\text { Irradiation area / } \\
\text { Kinoform size }\end{array}$ & \multicolumn{3}{|c}{0.249} \\
\hline Band-limited & \multicolumn{3}{|c}{} \\
\hline
\end{tabular}

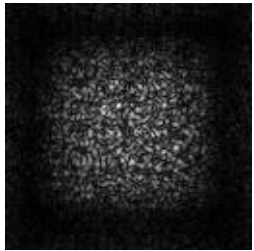

(B-1)

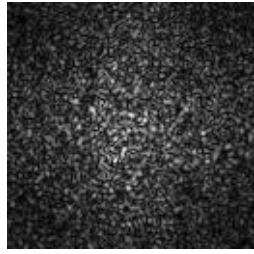

(B-2)

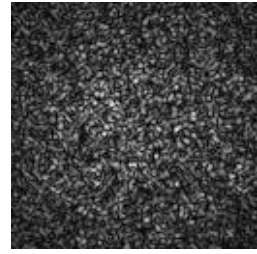

(B-3)
Fig.7. simulation $\mathrm{B}$ result 
Table4: Simulation C condition

\begin{tabular}{|l|c|c|c|}
\hline & C-1 & C-2 & C-3 \\
\hline Original Image & Fig.5(a) & Fig.5(b) & Fig.5(c) \\
\hline $\begin{array}{l}\text { Dummy area } \\
\text { ratio }\end{array}$ & $16 \times 16$ & $8 \times 8$ & $4 \times 4$ \\
\hline Kinoform of & \multicolumn{2}{|c|}{$1024 \times 1024[$ pixel] } \\
\hline $\begin{array}{l}\text { Number } \\
\text { lasers }\end{array}$ & $24 \times 24$ \\
\hline $\begin{array}{l}\text { Number of } \\
\text { iteration }\end{array}$ & 1000 times \\
\hline $\begin{array}{l}\text { Irradiation area / } \\
\text { Kinoform size }\end{array}$ & \multicolumn{2}{|c|}{0.249} \\
\hline Band-limited & \multicolumn{2}{|c|}{-} \\
\hline
\end{tabular}

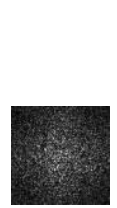

(C-1)

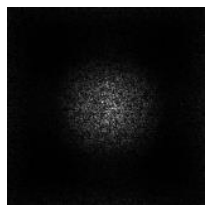

(C-2)

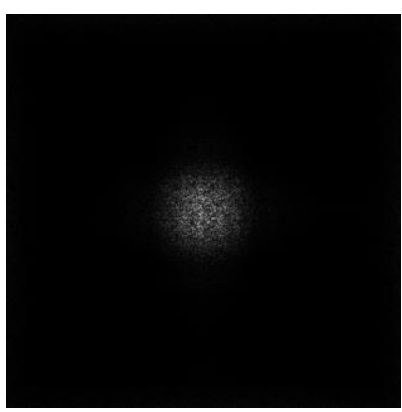

(C-3)
Fig.8. simulation $\mathrm{C}$ result

Table5: Simulation D condition

\begin{tabular}{|l|c|c|c|}
\hline & D-1 & D-2 & D-3 \\
\hline Original Image & \multicolumn{3}{|c|}{ Fig.5(a) } \\
\hline $\begin{array}{l}\text { Dummy area } \\
\text { ratio }\end{array}$ & $8 \times 8$ & $16 \times 16$ & $32 \times 32$ \\
\hline Kinoform & $\begin{array}{c}512 \times \\
512[\text { pixel] }\end{array}$ & $\begin{array}{c}1024 \times \\
1024[\text { pixel] }\end{array}$ & $\begin{array}{c}2048 \times \\
2048 \text { [pixel] }\end{array}$ \\
\hline $\begin{array}{l}\text { Number of } \\
\text { lasers }\end{array}$ & \multicolumn{3}{|c|}{$12 \times 12$} \\
\hline $\begin{array}{l}\text { Number of } \\
\text { iteration }\end{array}$ & 1000 \\
\hline $\begin{array}{l}\text { Irradiation area / } \\
\text { Kinoform ratio }\end{array}$ & 0.249 & 0.062 & 0.015 \\
\hline Band-limited & \multicolumn{3}{|c}{} \\
\hline
\end{tabular}

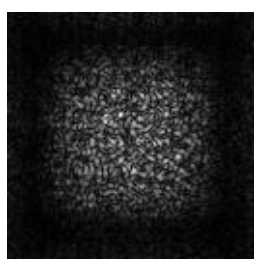

(D-1)

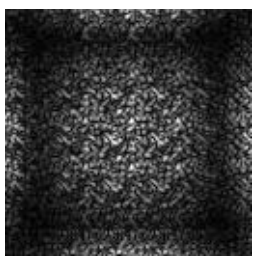

(D-2)

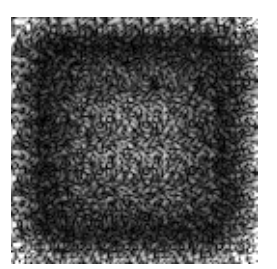

(D-3)
Fig.9. simulation D result
Table6: Simulation E condition

\begin{tabular}{|l|c|c|c|}
\hline & E-1 & E-2 & E-3 \\
\hline Original Image & Fig.5(c) & Fig.5(d) & Fig.5(e) \\
\hline $\begin{array}{l}\text { Dummy area } \\
\text { ratio }\end{array}$ & \multicolumn{2}{|c|}{$4 \times 4$} \\
\hline Kinoform of \\
\hline $\begin{array}{l}\text { Number of } \\
\text { lasers }\end{array}$ \\
\hline $\begin{array}{l}\text { Number of } \\
\text { iteration }\end{array}$ \\
\hline $\begin{array}{l}\text { Irradiation area / } \\
\text { Kinoform seize }\end{array}$ & $24 \times 24$ \\
\hline Band-limited & 0.249 \\
\hline
\end{tabular}

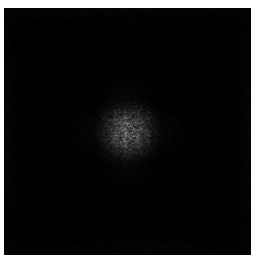

$(\mathrm{E}-1)$

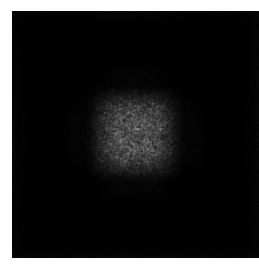

(E-2)

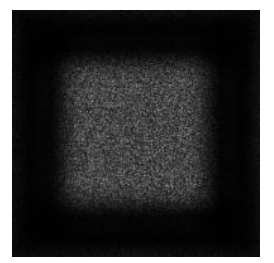

(E-3)
Fig.10. simulation E result

Figure 7(B-2), (B-3) show the influence of phase difference on beam shaping is big. Figure 7(B-1) shows the influence of phase difference on beam shaping is small. Two parameters of the number of laser lights and dummy area ratio are changed in simulation B. Therefore it can't be determined the influence of the number of laser lights.

We verify the influence of dummy area ratio to original image.Table4 shows simulation $\mathrm{C}$ condition of Kinoform generation. Figure 8 shows the reconstructed images of Kinoform of simulation $\mathrm{C}$ by multiple laser lights with phase difference. Figure 8 is the reconstructed images of the original image area only. In comparison of Figs 8 , the influence of phase difference on beam shaping is same level. Therefore simulation $\mathrm{B}, \mathrm{C}$ result shows the more the number of laser lights is large, the influence of phase difference on beam shaping is increased. Moreover simulation $\mathrm{B}, \mathrm{C}$ result shows dummy area ratio doesn't have relation of the influence of phase difference on beam shaping.

Next we verify the influence of irradiation area ratio to Kinoform size.Table5 shows simulation D condition of Kinoform generation. Figure 9 shows the reconstructed images of Kinoform of simulation D by multiple laser lights with phase difference. Figure 9 is the reconstructed images of the original image area only. Figure 9(D-2), (D-3) show the influence of phase difference on beam shaping is big. 
Figure 9(D-1) shows the influence of phase difference on

beam shaping is small. Simulation D result shows the more irradiation area ratio to Kinoform size is small, the influence of phase difference on beam shaping is increased.

Last we verify the influence of sampling points of focusing area.Table6 shows simulation $\mathrm{C}$ condition of Kinoform generation. Figure 10 shows the reconstructed images of Kinoform of simulation $\mathrm{C}$ by multiple laser lights with phase difference. Figure 10 is the reconstructed images of the original image area only. The edge of figs. 10(E-1), (E-2) is very dull. Figure 10(E-1), (E-2) show the influence of phase difference on beam shaping is big. Figure 10(E-3) shows the influence of phase difference on beam shaping is small. Simulation E result shows the more sampling points of focusing area is large, the more influence of phase difference on beam shaping is decreased.

\section{Conclusions}

We showed to be able to relax the influence of phase difference by using band-unlimited. We propose a new Kinoform design method using band-unlimited as the method for beam shaping of multiple laser diodes. This study showed the influence of phase difference on beam shaping of multiple laser lights are depended on the number of laser diodes, irradiation area ratio to Kinoform size and sampling points of the focusing area. It is necessary to use multiple laser diodes for laser machining and laser marking. However in the case of using many laser diodes, the influence on beam shaping is increased. From our simulation results, there are optimal parameters to obtain ideal reconstruction and it is possible to shape the light wavefront of multiple laser diodes to an arbitrary intensity distribution.

\section{References}

(1) H. Aagedal, M. Schmid, T. Beth, S. Teiwes, and F. Wyrowski: "Theory of speckles in diffractive optics and its application to beam shaping", J. Mod. Opt, No.43, 1409-1421, 1996

(2) Fred M. Dickey: "Laser beam shaping." Optics and photonics news, Vol.14, No.4, 30-35. 2003

(3) N. C. Gallagher and B. Liu: "Method for computing Kinoforms that reduces image reconstruction error", Applied optics Vol. 12, No. 10, 2328-2335, 1973

(4) L. B. Lesem, P. M. Hirsch, and J. A. Jordan: "The Kinoform: a new wavefront reconstruction device", IBM Journal of Research and Development, Vol. 13, No. 